\title{
Etablierung und Evaluation einer multinationalen Wissenschaftsakademie: Ein neues Modell zur Stärkung des wissenschaftlichen Nachwuchses in der mikrochirurgischen Forschung - Ein Konsensus Papier der DAM
}

\section{Establishing and evaluating a multinational Science Academy: A new tool for fostering scientific young talents in microsurgical research - a Consensus Statement of the DAM}

Autoren

R. E. Horch ${ }^{1}$, A. Kengelbach-Weigand', G. Pierer ${ }^{2}$, U. Kneser ${ }^{3}$, D. Schaefer ${ }^{4}$, A. M. Boos ${ }^{1,5}$, A. Arkudas ${ }^{1}$, V. J. Schmidt ${ }^{3}$

Institute

1 Plastisch- und Handchirurgische Klinik und Labor für Tissue Engineering und Regenerative Medizin, Universitätsklinikum Erlangen, Friedrich-Alexander Universität Erlangen-Nürnberg (FAU)

2 Universitätsklinik für Plastische, Rekonstruktive und Ästhetische Chirurgie, Tirol-Kliniken und der Medizinischen Universität Innsbruck

3 Klinik für Hand, Plastische und Rekonstruktive Chirurgie - Schwerbrandverletztenzentrum - BG - Unfallklinik Ludwigshafen, Klinik für Plastische Chirurgie der Ruprecht-Karls-Universität Heidelberg

4 Plastische, Rekonstruktive, Aesthetische und Handchirurgie, Universitätsspital Basel

5 Klinik für Plastische Chirurgie, Hand- und Verbrennungschirurgie, Universitätsklinikum Aachen

Schlüsselwörter

Mikrochirurgie, Replantation, Interdisziplinäre plastische Chirurgie, Wundheilung experimentell, Transplantation experimentell, Perfusion, Nervenregeneration, Mikrozirkulation

Key words

Microsurgery, replantation, interdisciplinary plastic surgery, wound healing, experimental, transplantation, perfusion, nerve regeneration, microcirculation

eingereicht 28.08.2019

akzeptiert 24.11.2019

Bibliografie

DOI https://doi.org/10.1055/a-1076-0933

Handchir Mikrochir Plast Chir 2020; 52: 140-146

(c) Georg Thieme Verlag KG Stuttgart · New York

ISSN 0722-1819
Korrespondenzadresse

Professor Dr. med. Raymund E Horch

Universitätsklinikum Erlangen Plastische

und Handchirurgische Klinik

Krankenhausstrasse 12

91054 Erlangen

Tel.: 09318533277

Fax: 0913139327

E-Mail: raymund.horch@uk-erlangen.de

\section{ZUSAMMENFASSUNG}

Hintergrund Die Bildung von beruflichen Netzwerken und Kooperationen ist neben einer guten Weiterbildung für eine erfolgreiche Karriere meist grundlegend. In etlichen Fachdisziplinen existieren hierfür bereits kleinere Symposien oder Konferenzen. Im Forschungszweig der Mikrochirurgie gab es bislang jedoch noch keine dezidierte Möglichkeit für Nachwuchswissenschaftler sich untereinander zu vernetzen.

Methoden Im Jahre 2017 wurde erstmalig eine Wissenschaftsakademie durch die Deutschsprachige Arbeitsgemeinschaft für Nerven und Gefäße (DAM) initiiert. Das Ziel war es, mikrochirurgisch forschende junge Ärzte sowie Wissenschaftler im kleinen Rahmen einmal jährlich zusammenzubringen und zu vernetzen, um Synergien für gemeinsame Forschungsprojekte zu entwickeln. Hierfür wurden jeweils von den Vorständen der Forschungseinrichtungen, die in der DAM organisiert sind, motivierte Nachwuchswissenschaftler zur Akademie entsendet. Nach einem Kennenlernen in lockerer Atmosphäre, bekamen die Teilnehmer die Möglichkeit, sich im Rahmen von Themenblöcken und unter Moderation durch erfahrene Mentoren ihr jeweiliges Forschungsprojekt vorzustellen. Es schloss sich eine Diskussionsrunde sowie Kleingruppenarbeit an, in der Kenntnisse und Methoden ausgetauscht sowie Anknüpfungspunkte für mögliche spätere Kooperationen identifiziert wurden.

Ergebnisse Die DAM Wissenschaftsakademie erwies sich in den vergangenen 3 Jahren als ein optimales Format, um Netzwerke von jungen mikrochirurgisch interessierten Ärzten und 
Wissenschaftlern zu initiieren und zu fördern. Es ergaben sich zahlreiche lebhafte und vertiefte Diskussionen, die vor allem durch die offene Arbeitsatmosphäre und die Verpflichtung zur Vertraulichkeit zustande kamen. Die meisten Synergien zeigten sich u. a. auf dem Gebiet Angiogenese, Bioreaktor, KarzinomADSC Interaktionen, Stammzellen, AV loop Modell, Ischämie/ Reperfusion und Nervenregeneration. Die Teilnehmer gaben in abschließender Evaluation durchweg ein sehr positives Feedback mit dem Wunsch der Fortsetzung dieser Akademie. Schlussfolgerung Die DAM Wissenschaftsakademie kann als eine sinnvolle Ergänzung zu den bereits bestehenden Möglichkeiten der Vernetzung unter mikrochirurgischen Forschern betrachtet werden. Die bisherigen Erfahrungen lassen annehmen, dass sich hieraus langfristige Kooperationen und ein dauerhafter Wissenstransfer unter den Teilnehmern ergeben.

\section{ABSTRACT}

Background The formation of professional networks and cooperations - in addition to any qualified good education - seems fundamental for a successful career. In a number of disciplines, various symposia or conferences exist. In the field of microsurgery, however, a specific, guided and designated opportunity for junior scientists to network with one another has been missing so far.

Methods In 2017, a science academy was initiated for the first time by the German-speaking Association for Nerves and Vessels (DAM) with the goal of bringing together and networking microsurgically researching young physicians and scientists.
This was intended to happen on a small scale once a year in order to develop synergies for joint research projects. For this purpose, motivated junior researchers were individually selected by their mentors and sent to the academy by the boards of research institutions that are organized in the DAM. After getting to know each other in a relaxed atmosphere, the participants were given the opportunity to present their respective research project within the framework of thematic blocks and moderated by experienced mentors. Each presentation was followed by a round table discussion and small group work, in which knowledge and methods were exchanged and points of contact for possible later cooperation were identified.

Results In the past 3 years, the DAM Science Academy proved to be an optimal format to initiate and promote networks of young researchers comprising microsurgically interested physicians and scientists. There were many lively and in-depth discussions, which were mainly due to the open working atmosphere and the obligation to confidentiality. Most of the synergies were shown i. a. in the field of angiogenesis, bioreactor, carcinoma-ADSC interactions, stem cells, AV loop model, ischemia/reperfusion, and nerve regeneration. The participants consistently gave a very positive feedback in the final evaluation with the wish to continue this academy.

Conclusion The DAM Science Academy can be considered a highly suitable complemental platform to the existing networking opportunities among microsurgical researchers. Experience so far suggests that this will hopefully result in long-term cooperations and a permanent transfer of knowledge among the participants.

\section{Einleitung}

Die Deutschsprachige Arbeitsgemeinschaft für Mikrochirurgie der peripheren Nerven und Gefäße (DAM) wurde anlässlich einer Handchirurgischen Tagung am 18.10.1977 in Erlangen gegründet. Vertreter der Länder Deutschland, Österreich und Schweiz erkannten seinerzeit den Bedarf einer sowohl standespolitischen als auch wissenschaftlichen Vertretung in diesem Zweig der Chirurgie, der zur damaligen Zeit als innovative Technik von immer mehr rekonstruktiv tätigen Chirurgen angewandt wurde. In dieser Pionierzeit verlief die Entwicklung der technischen Methoden, Innovationen und Verlässlichkeit der sogenannten Mikrochirurgie rasant. Es lag daher nahe, dass mit einer Bündelung gemeinsamer Aktivitäten auf diesem Feld nicht nur eine allgemein anerkannte Terminologie gefunden werden kann, sondern auch die Disziplin als solches weiterentwickelt und zu ihrer eigenen Profilierung und Verbreitung beitragen kann. Die neugegründete Arbeitsgemeinschaft hatte sich zum Ziel gesetzt, dieses neue Gebiet der Chirurgie nicht nur wissenschaftlich zu betreuen, sondern es war auch ein wesentlicher Gründungsgedanke, internationale Kontakte zum gezielten Wissenstransfer zu knüpfen und den Nachwuchs durch gezielten Unterricht zu fördern.

Das große Interesse an der Mikrochirurgie zeigte sich dann bereits im Gründungsjahr als 26 mikrochirurgisch interessierte Kollegen um eine ordentliche Mitgliedschaft nachsuchten. Seit 1978 wurden jährlich Tagungen in den beteiligten Ländern ausgerichtet, die allesamt durch regen Besuch und steigende Mitgliedszahlen gekennzeichnet waren. Die Bedeutung dieser Arbeitsgemeinschaft, welche ständig wächst und welche die mittlerweile größte Vereinigung auf dem Gebiet der Mikrochirurgie in Europa darstellt, lässt sich daran ablesen, dass die Expertengruppe bei vielen Rekonstruktiven Chirurgen als wesentliche Referenz für den mikrochirurgisch tätigen Arzt auf praktischem und wissenschaftlichem Gebiet gilt.

Die Förderung des wissenschaftlichen Nachwuchses war von Anfang an ein Kernanliegen der DAM. Anfänglich lobte die DAM Vortrags- und Wissenschaftspreisen sowie Reisestipendien aus. Dadurch sollten junge Mikrochirurgen im Ausland bei besonders renommierten Experten neueste Operationstechniken erlernen.

Mittlerweile wurden mikrochirurgische Operationsverfahren und Lappentechniken weltweit jedoch zunehmend standardisiert und dürfen heute trotz der ohne Zweifel oftmals gegebenen Komplexität nicht mehr als spektakuläre Einzelaktionen, sondern vielmehr als Teil der klinischen Versorgungsroutine mit hohen Erfolgsraten in spezialisierten Zentren angesehen werden. Vor diesem Hintergrund hatte die DAM neben der Einrichtung einer jährlich stattfindenden DAM Fortbildungsakademie auch die Etablierung einer DAM Wissenschaftsakademie zum Wissensaustausch und zur Vernetzung junger mikrochirurgisch aktiver ärztlicher und na- 


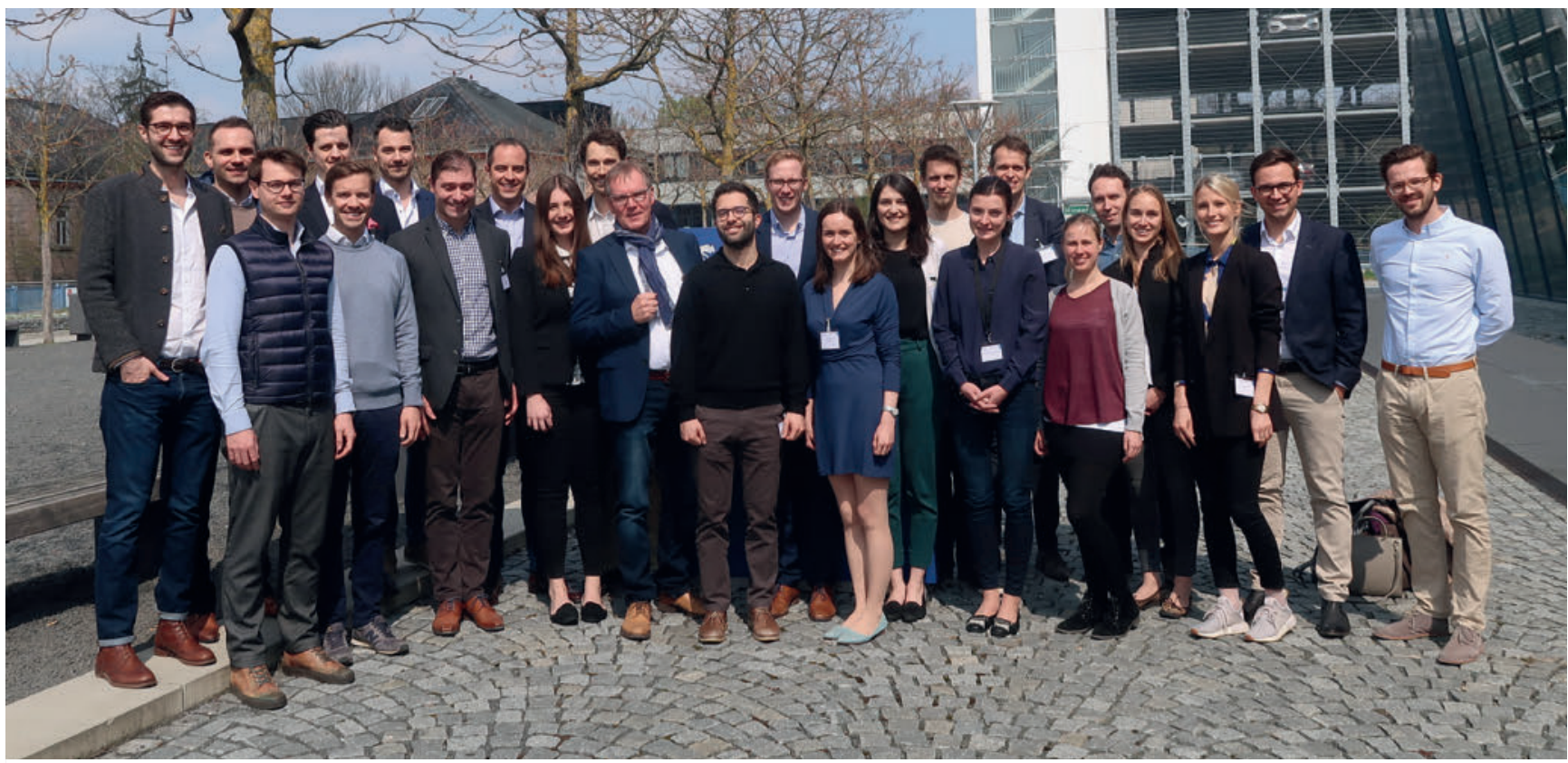

- Abb. 1 Bild der Teilnehmer DAM Wissenschaftsakademie 2019.

turwissenschaftlicher Forscher beschlossen und im Jahr 2017 gestartet.

Im Folgenden soll über die Erfahrungen mit der Etablierung dieser neuen modellartigen Wissenschaftsakademie berichtet werden.

\section{Methodik und Rahmenbedingungen}

Von den Vorständen der Forschungseinrichtungen, die in der DAM organisiert sind, erhielt jeder die Möglichkeit einen als geeignet erscheinenden Mitarbeiter zur jährlichen Akademie zu entsenden. Diese Vorgehensweise ist analog zu der ebenfalls von der DAM jährlich ausgerichteten mikrochirurgischen Weiterbildungsakademie [1]. Damit sollten auch die jeweiligen Forschungsschwerpunkte der Institutionen repräsentiert werden, die von den Zuständigen als relevant und zukunftsfähig eingeschätzt wurden. Das Prinzip einer Vorselektion und Konzentration der Themen wurde dabei bewusst ausprobiert. Das Konzept stellte sich im Verlauf der ersten 3 Jahre als äußerst zielführend heraus, da sich wesentliche Forschungsrichtungen herauskristallisierten, die letztlich auch das Spektrum des wissenschaftlichen Engagements der DAM widerspiegeln.

Nach einem gemeinsamen Kennenlernen am Vorabend der Akademie in lockerer Atmosphäre in Erlangen und Vorstellungsrunde der Teilnehmer, wurden zunächst die eigenen Projekte geordnet nach Themenblöcken dem Publikum präsentiert. Im Anschluss daran fand eine intensive Kleingruppenarbeit und Diskussion statt mit dem Ziel, Kenntnisse auszutauschen, gemeinsame Herausforderungen in der Forschung zu identifizieren, und Anknüpfungspunkte für gemeinsame Aktivitäten und den wissenschaftlichen Austausch zu definieren. Nachfolgend wurden die erarbeiteten Themen im Plenum vorgestellt und gemeinsam diskutiert. Ein aus der Gruppe benannter Protokollführer erstellte eine Zusammenfassung des jeweiligen Themenblocks und der Diskussionen in einer
Berichtsform für den DAM Vorstand, der für die Darstellung auf der DAM Homepage gedacht ist.

Die nachfolgende Tabelle gibt eine Übersicht über die konkret behandelten Themen der verschiedenen Nachwuchsforscher in den Jahren 2017, 2018 und 2019. Dabei wird die Vielfalt und auch die aktuelle wissenschaftliche Ausrichtung der in der DAM organisierten aktiven Plastisch-Chirurgischen Einrichtungen mit Forschungsauftrag erkennbar.

Im Anschluss an die jeweiligen Vortragsblöcke fanden eine detaillierte Besprechung und ergebnisoffene Diskussionen der präsentierten Methoden und Ergebnisse statt. Für diese Diskussionen wurde bewusst ein großes, aber flexibles Zeitfenster eingeplant, um die Möglichkeit zu schaffen, auf den jeweiligen Diskussionsbedarf eines Themenblocks ausreichend eingehen zu können. Ein wesentliches Ziel war hierbei, methodische und inhaltliche Synergien zwischen den verschiedenen Institutionen zu finden, um standortübergreifend wissenschaftliche Ressourcen zu nutzen und Exzellenzfunktionen von einzelnen Einrichtungen (z. B. Methoden wie das „Single-Cell Sorting“ oder die AV Loop Technik zur Gewebezüchtung, Austausch von Zell-Linien etc.) weiteren motivierten Kollegen/innen aus dem deutschsprachigen Raum zugänglich zu machen. Bewusst sollte damit das Schaffen von standortübergreifenden Forschungskooperationen ermöglicht und gefördert werden.

Dies soll ebenso dazu dienen, das Fachgebiet der Plastischen und Handchirurgie mit der Mikrochirurgie insgesamt gegenüber den größeren chirurgischen „Kernfächern“, welche aktuell noch immer über zahlenmäßig deutlich mehr universitären Vertretungen verfügen, wissenschaftlich konkurrenzfähiger zu machen. Giunta et al haben mehrfach darauf hingewiesen, wie wichtig eine starke universitäre Repräsentanz hierfür ist, auch im Hinblick auf die Beurteilung von Forschungsanträgen durch die Deutsche Forschungsgemeinschaft DFG [2]. Eine Evaluation der Arbeitstagung unterstützt die Eckpunkte der jeweils erreichten Arbeitsprogramme und gemeinsamen künftigen Forschungsprojekte der Teilnehmer. 
> Tab. 1 Vortragsthemen der DAM Wissenschaftsakademie 2017-2019.

\section{Gruppe 1: Angiogenese}

Etablierung AV loop Modell als Tumorangiogenese-Modell

Endotheliales Connexin43 als angioinduktiver Effektor

Intrinsische Vaskularisation von Biomaterialien im Kleintier

\section{Gruppe 2: Regenerative Medizin}

Bioreaktorsystem zum Aufbau eines vaskularisierten Gewebes

Skelettmuskel Tissue Engineering auf PCL-Kollagen-Nanofaserscaffolds im mikrochirurgischen Gefäßschleifenmodell

Engineering of axially-vascularized osteogenic grafts for the treatment of avascular necrosis of bone

Axial vaskularisiertes Knochen Tissue Engineering im Großtiermodell

\section{Gruppe 3: Klinische mikrochirurgische Forschung}

Ist Formalin-fixiertes Gewebe geeignetes Übungsmaterial für mikrochirurgische Zwecke?

Perioperatives Management bei DIEP-Lappenplastik

Murine und humane ADSC aus unterschiedlichen Isolationsmethoden zur klinischen Anwendung

\section{Gruppe 4: Ischämie/Reperfusion}

Acellular Hypothermic Extracorporeal Perfusion Extends Allowable Ischemia Time in a Porcine Whole Limb Replantation Model

Therapeutisches Targeting von C-reaktivem Protein reduziert den Ischämie/Reperfusionsschaden in der Mikrochirurgie

Immunologische Untersuchungen der allogenen Abstoßungsreaktion im Hinterlaufmodell der Ratte

Extrakorporale Perfusion und Oxygenierung von Muskellappen zur Verringerung von Ischämie-induziertem Schaden

2018 Gruppe 1: Lymphgefäßsystem - Mikrochirurgische Forschung

Deckung von kritischen Defekten durch eine hämodynamisch stimulierte und intrinsisch vaskularisierte Bindegewebslappenplastik im Klein- und Großtiermodell

Tissue engineering of lymphatic vasculature in vivo

Etablierung eines standardisierten und validierten Kleintiermodels zur Untersuchung des mikrochirurgisch induzierten Lymphödems Standardisiertes Konzept und optimiertes nie mit bei der chirurgischen Behandlung von Lymphödemen.

Analyse des lymphangiogenen Potentials vaskularisierter Lymphknotenlappen in einem axillären Ischämie-Reperfusions-Modell an Ratten

\section{Gruppe 2: Ischämie - Reperfusion - Entzündung}

Die Interaktion von C-reaktivem Protein mit dem Komplementsystem in der Entzündungsantwort

Minimal-invasiver Nachweis von Zytokinen zur Diagnose einer Abstoßungsreaktion

Optimierung der Mikrozirkulation - Einsatz von Remote Ischemic Conditioning (RIC) in der Plastischen Chirurgie

\section{Gruppe 3: Muskuloskelettales Tissue Engineering - Rekonstruktion}

Generierung von bioartifiziellen Knochenersatzgeweben mit ADSCs und HUVECs

Engineered, axially-vascularized osteogenic grafts from human adipose-derived cells to treat avascular necrosis of bone

Hypertrophic cartilage - a treatment for complex bone defects

Pedikuläre Dezellularisierung und Rezellularisierung eines kompletten Skelettmuskels

Spontane Resensibilisierung von freien Lappenplastiken in der Rekonstruktion der unteren Extremität

\section{Vorträge Gruppe 4: Mikrochirurgie}

Hochleistungskeramik-Beschichtungen chirurgischer Instrumente zur Optimierung mikrochirurgischer Techniken

Erfahrungen und Studien aus unserem Mikrochirurgie-Kursen

Vorträge Gruppe 5: Regenerative Medizin - Vaskularisation

Etablierung eines intravitalmikroskopischen Modells zur Analyse der leukozytär vermittelten zellulären Mechanismen bei der de novo Gewebsformierung im AV-Loop Modell an der Ratte

Fluss-induzierte Stimulation der Angiogenese durch miRNAs und Gene in arteriovenösen Loops: Vom Rattenmodell zum Menschen

Integrin alpha $V$ und Integrin alpha 5 regulieren die Proliferation und

Differenzierung von humanen adipozytären Vorläuferzellen

Entwicklung eines axial gestielten Lipotransplantates im Kleintiermodell

2019 Gruppe 1: Fettgewebe und Tumor

Etablierung eines arteriovenösen Gefäßschleifenmodells für die Untersuchung der Interaktion von ADSC und Mammakarzinomzellen

Onkologische Sicherheit autologer fettgewebsbasierter Brustrekonstruktionsverfahren und ihr Einfluss auf die „tumor dormancy“ im vergleichenden Kleintiermodell

Der Einfluss extrazellulärer Komponenten auf die Physiologie von menschlichen Fettstammzellen 
- Tab. 1 Fortsetzung.

Analyse der Tumor-Dormancy und Entwicklung in biofabrizierten und in-vivo-vaskularisierten 3D-Modellen

Veränderte Gewebemorphologie, ein ausgeprägtes biochemisches Profil und das Fehlen eines lymphatischen Phänotyps liegt Lipödem zugrunde

\section{Gruppe 2: Tissue Engineering und Vaskularisation}

Die Extrazellulärmatrix in der Interaktion zwischen Osteoblasten und Endothelzellen und deren Bedeutung für das Tissue Engineering des Knochens

Die temporäre Abgabe von fibringebundenen VEGF-Protein in osteogenen Transplantaten führt sowohl zu einer gesteigerten Vaskularisation als auch einer effizienten Knochenbildung

Spheroidbidung und Modulation der tenoszytenspezifischen Genexpression unter stimulierter Mikrogravitation

Gruppe 3: Nervenregeneration

Der Effekt von ANISOGEL auf die periphere Nervenregeneration nach Läsion des nervus ischiadicus in der Ratte

Regenerationsergebnisse nach Rekonstruktion sensibler digitaler Nervendefekte der Hand mittels Venen-Muskel-Interponaten

Von der Rekonstruktion zum Neurom - Experimentelle Strategien mit Kollagen und Chitosan zur Behandlung von peripheren Nervenverletzung

Nerventransfer

Selektiver Nerventransfer zur Rekonstruktion von Nervus peroneus Läsionen - eine klinisch-anatomische Studie

\section{Gruppe 4: Entzündung/neue Therapieansätze}

Die pharmazeutische Modulation des angeborenen Immunsystems als therapeutischer Ansatz der post-chirurgischen Entzündungsantwort und allogenen Transplantatabstoßung

Biofabrikation zellularisierter und im AV Loop vaskularisierter Gewebecontainer für die Transplantation wirkstoffproduzierender Zellen

Therapie von Wirbelkörperdefekten im Rattenmodell - Modelletablierung und Testung verschiedener Implantmaterialien

Gruppe 5: Lappenplastiken/Monitoring

Perfusions-Kartierung bei gestielten Lappenplastiken

Negativdruck-induzierte Hyperämie zum Monitoring freier Lappenplastiken

Katecholamine in der Mikrochirurgie - Perfusionsmonitoring beim Deep Inferior Epigastric Perforator Flap

\section{Diskussion}

Versuche, die akademische Karriere von jungen Talenten vor dem Hintergrund einer sich derzeit immer mehr verdichtenden klinischen Arbeitsbelastung und einem regulatorisch enger werdenden Arbeitszeitrahmen zu fördern, wurden in der Literatur auf unterschiedliche Weise, meistens jedoch in einzelnen Institutionen unternommen [3-6]. Auf die Notwendigkeit ebenso wie auf die Problematik einer langfristigen Förderung des akademischen Nachwuchses wurde mehrfach hingewiesen [7-9]. Nachdem das Fachgebiet Plastische Chirurgie in den 1990er Jahren relativ spät seine eigene Facharztidentität erhielt, wuchs auch die Zahl der akademischen Vertretungen an Universitätskliniken kontinuierlich [2, 10]. Im Bereich der relevanten Forschungsförderungen spiegelt sich diese zunehmend breitere wissenschaftliche Basis ebenfalls wider $[2,11]$. Trotz beachtlicher Anstrengungen zur Nachwuchsförderung einzelner Zentren wurde schon früh darauf aufmerksam gemacht, dass für eine höhere wissenschaftliche Qualität und Quantität sowie für eine starke Forschungsprofilierung als Gesamtfach letztlich langfristige gebündelte Anstrengungen mehrerer oder vieler Zentren notwendig erscheinen [12]. In der Literatur gilt es dabei als unbestritten, dass die Qualität der Mentoren eine wesentliche Rolle für die langfristige Ausrichtung und die Ausbildung spielt [9, 13]. Für die praktische Ausbildung in der Mikrochirurgie existieren schon lange valide Bemühungen, diese zu einem festen Bestandteil der mikrochirurgischen Entwicklung zu machen und zu optimieren [14-24]. Für die wissenschaftliche Ausrichtung junger sog. „High Potentials“ in der Mikrochirurgischen Forschung wurde bisher je- doch keine vergleichbar koordinierte multizentrische Anstrengung unternommen. Im Gegensatz hierzu sind internationale Veranstaltungen wie z. B. das Plastic Surgery Research Council vom Grundsatz und von der Zielsetzung her ähnlich, jedoch im Format etwas anders ausgelegt $[25,26]$. Sie dienen zwar ebenfalls wie andere Wissenschaftsveranstaltungen der Vernetzung und greifen Themen wie Weiterbildung, Aspekte einer wissenschaftlichen Karriere einschließlich Tutorien und Workshops auf [27, 28]. Sie bestehen zur Hälfte aus rein klinischen Fallserien und Fragestellungen und geben jeweils einen Überblick über die Fülle an Forschungsthemen in der Plastischen Chirurgie. Sie umfassen somit das gesamte Spektrum aller möglichen Forschungsrichtungen in der Chirurgie, gleichen aber in der praktischen Durchführung mit extrem eng getakteten und daher möglichst ausformulierten Vorträgen und minimaler Diskussionszeit eher dem klassischen Kongressformat.

Nach den entsprechenden thematischen und organisatorischen Vorbereitungen konnte erstmals im Jahr 2017 in Erlangen eine DAM-Wissenschaftsakademie stattfinden. Das Hauptziel bestand dabei in einer Netzwerkbildung und Förderung der experimentellen und klinischen Forschung in der Mikrochirurgie. Der Schwerpunkt der Forschungsfelder sollte an die relevanten Themen der DAM angelehnt bleiben. Dabei war es das Anliegen, dass nach Möglichkeit junge Projektteilnehmer mit der langfristigen Vision einer akademisch-chirurgischen Karriere und bereits gestarteten Projekten einmal im Jahr zusammenkommen. Es zeigte sich, dass das Ziel der in der Tradition der DAM erprobten offenen konstruktiven Diskussion von Forschungsansätzen unterschiedlicher Richtungen und 
die gegenseitige Unterstützung und Förderung von Forschungsprojekten auf dem Gebiert der Mikrochirurgie von den Teilnehmern äußerst gut umgesetzt wurde. Erfahrene Mentoren, die von der DAM benannt wurden, sollten zum Beispiel auch in Hinblick auf spätere selbständige Kongressorganisationen im größeren Rahmen die Planung und Durchführung der Tagung organisieren und unter Anleitung eines Vorstandsmitglieds der DAM leiten. Auch dieses Ziel wurde ohne Zweifel erreicht. Ein offener konstruktiver Geist mit einem standortübergreifenden Gruppengefühl herrschte nach allgemeiner Auffassung aller Teilnehmenden zu jeder Zeit vor. Die meisten klassischen Wissenschaftsveranstaltungen bestehen nach Lingner heute aus einer strikt durchgetakteten Aufeinanderfolge von vielen möglichst gleichlangen Vorträgen in einem möglichst knapp kalkulierten Zeitrahmen mit einer oft zu kurz bemessenen Diskussionszeit [29]. Diese wird häufig aus Zeitgründen auf ein absolutes Minimum reduziert. Damit gewinnen etliche wissenschaftliche Symposien und Kongresse den „Charakter einer Art Casting Show für den akademischen Karriereweg und ggf. für den akademischen Stellenmarkt“ " 29]. Häufig entsteht bei solchen größeren Veranstaltungen der Eindruck, dass dabei letztlich oft nicht mehr als ein konzentriertes Vorlesen von bereits bestens ausformulierten Aufsätzen abläuft und allenfalls in der knapp bemessenen Diskussionszeit noch mehr oder weniger kurze Verständnisfragen aus dem Publikum möglich sind. Für den akademischen Nachwuchs besteht daher in diesen Formaten die Gefahr, sich gar nicht mehr in Rede und Gegenrede argumentativ positionieren zu müssen. Das geht auch mit einem gewissen Risiko einher, dass der wissenschaftliche Nachwuchs die Konformität als für das eigene Fortkommen vorteilhaft ansehen könnte. Im Gegenteil sollte aber eine fachlich fundierte Diskussionskultur der Normalfall sein, um den Erkenntnisprozess für den Einzelnen und die Fachdisziplin voranzutreiben. Nachdem im Zuge des Konkurrenzkampfes um Mittelzuweisungen auch in der akademischen Welt die möglichst breite mediale Vermarktung von Veranstaltungen bereits als eigenes Erfolgskriterium angesehen wird, besteht daher aus Sicht der DAM umso mehr die Notwendigkeit spezieller Formate für die wissenschaftliche Weiterentwicklung der Disziplin. Daher wurde das oben beschriebene Konzept einer Wissenschaftsakademie etabliert und anhand der ersten 3 Jahre diese Form des Wissenschaftsaustausches und der Vernetzung evaluiert. Die dadurch gewünschte Abgrenzung von durchaus ebenfalls wichtigen und interessanten Formaten anderer Fachgesellschaften, wie z. B. diverse Forschungstage oder internationale Research Councils etc., zeichnete die bisherigen Tagungen der DAM Wissenschaftsakademie nachhaltig aus und hat sich wegen der sehr produktiven Arbeitsatmosphäre bewährt. Der Wunsch aller Teilnehmer, diese neue Form der Wissenschaftsakademie unbedingt fortzusetzen spricht auch für den subjektiven Benefit der jungen Forscher innerhalb der DAM.

Die Frage, ob es Anknüpfungspunkte für Vernetzungen innerhalb der mikrochirurgischen Forschergemeinschaft gibt, und wo man Synergien nutzen kann stand bei allen Veranstaltungen als Leitgedanken im Vordergrund der Arbeitstagung. Es entwickelten sich in der Folge jeweils konkrete Wissenstransfers und Methodenaustausch zwischen den Forschergruppen, wobei sich hier die meisten Anknüpfungspunkte bei den Themen Angiogenese/Bioreaktor/Karzinom - ADSC Interaktion/Stammzellen/AV-loop Modell/ Ischämie - Reperfusion und Nervenregeneration ergaben. Im Re- sümee zeigte sich auf jeden Fall, dass die Diskussionen wesentlich lebhafter als auf Kongressen üblich verliefen und diese spezielle offene Arbeitsatmosphäre als sehr positiv eingestuft wurde. Nachdem sich alle Teilnehmer zur absoluten Vertraulichkeit verpflichtet hatten, wurden bewusst auch unpublizierte Daten oder noch im Entstehen befindliche Forschungsansätze präsentiert, die dann ebenso offen diskutiert werden konnten. Auch dieser Aspekt wurde positiv angenommen.

In der Zusammenfassung kann man somit festhalten, dass die Etablierung einer DAM Wissenschaftsakademie als Ergänzung zu anderen eher klassischen Formaten der Forschungszusammenkünfte ein neues und interessantes alternatives Modell sein kann, welches eine langfristig nachhaltige Vernetzung junger Forscher, einen dauerhaften Wissenstransfer auf persönlicher Ebene und Generierung von Synergieeffekten darstellt und ggf. auch als Modell für andere Fachgesellschaften dienen könnte.

\section{Interessenkonflikt}

Die Autoren geben an, dass kein Interessenkonflikt besteht.

\section{Literatur}

[1] Bergmeister KD, Kneser U, Kremer T et al. New concept for microsurgical education: The training academy of the German Working Group for Microsurgery of Peripheral Nerves and Vessels: Results of a 4-year evaluation. Handchir Mikrochir Plast Chir 2019; 51: 327-333

[2] Giunta RE, Mollhoff N, Horch RE et al. Registry Research Funding of the German Society of Plastic, Reconstructive and Aesthetic Surgeons (DGPRAC) and Research Funding Report 2017/2018. Handchir Mikrochir Plast Chir 2018; 50: 414-421

[3] Kelley WN, Stross JK. Faculty tracks and academic success. Ann Intern Med 1992; 116 (8): 654-9

[4] Goldie J, Dowie A, Goldie A et al. What makes a good clinical student and teacher? An exploratory study. BMC Med Educ 2015; 15: 40

[5] Pardamean B. Measuring change in critical thinking skills of dental students educated in a PBL curriculum. J Dent Educ 2012; 76 (4): 443-53

[6] Kemper R, Horch RE, Giunta RE et al. Interdisciplinarity in German academic Plastic Surgery - irrelevant or forward-looking? Handchir Mikrochir Plast Chir 2019; 51: 144-150

[7] Lehnhardt M, Kneser U, Giunta R et al. Commentary on the Paper: The Humboldtian model in Plastic and Reconstructive Surgery - a student survey on teaching and venia legendi. Handchir Mikrochir Plast Chir 2018; 50 (2): 140-141

[8] Kolbenschlag J, Paprottka F, Wagner J et al. Quo vadis DGPRAC: associated members' goals for the future. Handchir Mikrochir Plast Chir 2017; 49 (4): 267-272

[9] Horch RE, Vogt PM, Schaller HE et al. Strategies to ensure careers of young academics in plastic surgery - analysis of the current situation and future perspectives. Handchir Mikrochir Plast Chir 2013; 45 (4): 193-9

[10] Kemper R, Horch RE, Giunta RE et al. Interdisciplinarity in German academic Plastic Surgery - irrelevant or forward-looking? Handchir Mikrochir Plast Chir 2019; 51 (2): 144-150

[11] Giunta RE, Horch RE, Prantl L et al. Registry Research Funding of the German Society of Plastic. Reconstructive and Aesthetic Surgeons 
(DGPRAC) and Research Funding Report 2015/2016. Handchir Mikrochir Plast Chir 2016; 48 (6): 370-373

[12] Miller SH. Competitive forces and academic plastic surgery. Plast Reconstr Surg 1998; 101 (5): 1389-99

[13] Chen JT, Girotto JA, Kitzmiller WJ et al. Academic plastic surgery: faculty recruitment and retention. Plast Reconstr Surg 2014; 133 (3): $393 e-404 e$

[14] Schaverien MV, Butler CE, Suami H et al. Interview Scores Correlate with Fellow Microsurgical Skill and Performance. J Reconstr Microsurg 2018; 34 (3): 211-217

[15] Pafitanis G, Narushima M, Yamamoto T et al. Evolution of an evidencebased supermicrosurgery simulation training curriculum: A systematic review. J Plast Reconstr Aesthet Surg 2018; 71 (7): 976-988

[16] Klein H]. The International Fellowship Program in Microsurgery at Uppsala University Hospital: A Paragon Throughout Europe. Ann Plast Surg 2018; 81 (2): 137-139

[17] Pafitanis G, Cooper L, Vijayan R et al. Putting the heart into microvascular training: The micropump, a practical "heart-like“ device to enhance vascular anastomosis non-living simulation. J Plast Reconstr Aesthet Surg 2017; 70 (12): 1787-1788

[18] Mason KA, Theodorakopoulou E, Pafitanis G et al. Twelve tips for postgraduate or undergraduate medics building a basic microsurgery simulation training course. Med Teach 2016; 38 (9): 872-8

[19] Ghanem AM, Al Omran Y, Shatta B et al. Anastomosis Lapse Index (ALI): A Validated End Product Assessment Tool for Simulation Microsurgery Training. J Reconstr Microsurg 2016; 32 (3): 233-41

[20] Beth Grossman L, Komatsu DE, Badalamente MA et al. Microsurgical Simulation Exercise for Surgical Training. J Surg Educ 2016; 73 (1): 116-20

[21] Singh M, Ziolkowski N, Ramachandran S et al. Development of a fiveday basic microsurgery simulation training course: a cost analysis. Arch Plast Surg 2014; 41 (3): 213-7

[22] Myers SR, Froschauer S, Akelina $Y$ et al. Microsurgery training for the twenty-first century. Arch Plast Surg 2013; 40 (4): 302-3

[23] Selber JC, Chang El, Liu J et al. Tracking the learning curve in microsurgical skill acquisition. Plast Reconstr Surg 2012; 130 (4): 550e-557e

[24] Neaman KC, Hill BC, Ebner B et al. Plastic surgery chief resident clinics: the current state of affairs. Plast Reconstr Surg 2010; 126 (2): 626-33

[25] Noone RB, Goldwyn RM, McGrath M et al. 50th Anniversary Plastic Surgery Research Council Panel on the Future of Academic Plastic Surgery. Plast Reconstr Surg 2007; 120 (6): 1709-21

[26] Rohrich RJ. The Seventh Annual Meeting of the European Plastic Surgery Research Council. Plast Reconstr Surg 2015; 136 (2): 434-5

[27] Gosain AK, Purnell CA, Sweeney WS. Mentorship in Research. Plast Reconstr Surg Glob Open 2015; 3 (3): e363

[28] Gast KM, Kuzon WM Jr., Adelman EE et al. Influence of training institution on academic affiliation and productivity among plastic surgery faculty in the United States. Plast Reconstr Surg 2014; 134 (3): 570-8

[29] Lingner M. Dabeisein ist alles? Gedanken zum akademischen Tagungs(un)wesen. Forschung und Lehre 2019; 26 (5): 450-451 